\title{
Bolometric ferromagnetic resonance techniques for characterising spin-Hall effect at high temperatures
}

\author{
P. Phu, ${ }^{1}$ K. Yamanoi,${ }^{1,2}$ K. Ohnishi, ${ }^{2,3}$ J. Hyodo,${ }^{4}$ K. Rogdakis,${ }^{1}$ \\ Y. Yamazaki, ${ }^{4,5,6}$ T. Kimura, ${ }^{2,3}$ and H. Kurebayashi ${ }^{1}$ \\ ${ }^{1}$ London Centre for Nanotechnology, \\ University College London, 17-19 Gordon Street, \\ London, WC1H OAH, United Kingdom \\ ${ }^{2}$ Department of Physics, Kyushu University, \\ 744 Motooka, Fukuoka 819-0395, Japan \\ ${ }^{3}$ Research Center for Quantum Nano-Spin Sciences, \\ Kyushu University, 744 Motooka, Fukuoka 819-0395, Japan \\ ${ }^{4}$ INAMORI Frontier Research Center, \\ Kyushu University, Fukuoka 819-0395, Japan \\ ${ }^{5}$ Kyushu University Platform of Inter-/Transdisciplinary Energy Research, \\ Kyushu University, 744 Motooka, Nishi-ku, Fukuoka 819-0395, Japan \\ ${ }^{6}$ Department of Materials Science and Engineering, \\ Graduate School of Engineering, Kyushu University, \\ 744 Motooka, Nishi-ku, Fukuoka 819-0395, Japan
}

(Dated: April 4, 2019)

Keywords: Magnetization dynamics; Spin-transfer-torque; Spin-Hall-angle, High-temperature-measurement 
We report on current-induced ferromagnetic resonance techniques to characterise spin-Hall effect at high temperatures. A microwave current was injected into a patterned $\mathrm{CoFeB} / \mathrm{Pt}$ bi-layer grown on a glass substrate, simultaneously exerting spin-transfer torques through the spin-Hall effect and also causing Joule heating enabling the control of the device temperature. We measured the device temperature by using the device itself as a local temperature sensor. A clear reduction of $\mathrm{CoFeB}$ magnetisation was observed as the device temperature was increased allowing us to estimate the Curie temperature of our CoFeB film to be $920 \mathrm{~K}$. The spin-Hall angle of $\mathrm{Pt}$ was quantified as $(1.72 \pm 0.03) \times 10^{-2}$ at $300 \mathrm{~K}$ and was slightly increased to $(1.75 \pm 0.02) \times 10^{-2}$ at $410 \mathrm{~K}$. This simple method can be widely used for quantifying the spin-Hall angle of a large variety of materials at high temperatures.

\section{INTRODUCTION}

Spin currents ${ }^{1}$, a flow of spin angular momentum without any charge transfer, have been attracting a great deal of interests as a building block of current and future spintronic devices. To find efficient ways of electrically generating and detecting spin currents is a central aim, thereby enabling manipulation of magnetic states at a small electric power consumption. This is directly relevant to spintronic memory device developments, or magnetic RAM (MRAM) technologies, where optimisation of conventional spin-transfer torques ${ }^{2}$ has reached a certain level of maturity owing to intensive efforts during the last few decades ${ }^{3}$. The spinHall effect (SHE) which arises from the spin-orbit interaction in solid states materials has emerged as a future alternative towards this line of research. Liu et al. demonstrated that SHE in heavy metals such as Ta can switch a sub-micron meter magnetic memory cell ${ }^{4}$ with an electric current amplitude similar to what is required for a conventional spin-transfer torque switching. Stimulated by this work and other earlier reports on spin-orbit torques ${ }^{5-9}$, scientists have been investigating $\mathrm{SHE}^{10}$ on different materials system aiming the optimization of the effect. A figure of merit for SHE is the spin-Hall angle $\left(\theta_{\mathrm{SH}}\right)$ that is essentially the ratio between diagonal and off-diagonal components in the conductivity tensor. Some heavy metals show a large $\theta_{\mathrm{SH}}$ greater than $0.01^{4,11,13}$, primarily because of the high atomistic spin-orbit coupling strength. In addition, a small amount of heavy element doping in 
$\mathrm{Cu}$ was found to be very effective in increasing $\theta_{\mathrm{SH}}$ via the extrinsic SHE mechanism ${ }^{14}$. The effective $\theta_{\mathrm{SH}}$ in topological insulators have also been studied, with some cases showing $\theta_{\mathrm{SH}}$ much greater than the unity ${ }^{15,16}$.

Characterising the influence of spintronic effects such as the SHE at high temperature will be increasingly important at the device application regime. For instance, current-induced magnetisation switching in a single MRAM cell requires a large current that causes a local and temporal temperature rise. This can potentially lead to device performance being affected during the switching. It is therefore useful to have a tool to characterise SHE at a high temperature regime. Measurement techniques of SHE include spin-pumping ${ }^{7}$, spin-transfer ferromagnetic resonance $(\mathrm{ST}-\mathrm{FMR})^{12}$, spin-absorption in lateral spin-valves ${ }^{17-19}$ and optical spin orientation ${ }^{20}$. Some of these techniques have been exploited to study temperature dependence of $\theta_{\mathrm{SH}}{ }^{18,23}$. However, the temperature range of these previous reports only covers below room temperature (RT) and SHE at higher temperatures has been largely overlooked. This could be due to the fact that spintronic research groups across the world mostly possess cryogenic systems for low-temperature studies, whereas, for high temperatures, there have been little efforts in integrating a heating function as well as introducing an accurate temperature sensor near a lithographically patterned device. Incorporating all of these within an area between magnet poles (typically a few cm wide for electromagnets) is also non-trivial.

In this paper, we report on a simple method of characterising SHE at high temperatures. It is based on the combination of ST-FMR techniques and the use of devices as a local temperature sensor. Based on this approach, microwave currents through a device generate effective spin torques for driving FMR allowing to quantify their magnetic properties and $\theta_{\mathrm{SH}}$. At the same time, we use the same device to extract the actual temperature while injecting microwaves at different excitation powers. This is possible by measuring the device resistance for each microwave power and by comparing it with the temperature dependence of the device resistance we perform separately. This paper shows the proof of principle of this proposed method by using a standard bi-layer of $\mathrm{CoFeB} / \mathrm{Pt}$ for characterising SHE at high temperatures. We are able to discuss a reduction of magnetisation with increasing temperature, from which the Curie temperature of the CoFeB film has been deduced. $\theta_{\mathrm{SH}}$ of $\mathrm{Pt}$ at high temperatures have been also quantified and we compare our results with other studies that present the temperature dependence of Pt's $\theta_{\mathrm{SH}}$ below RT. 


\section{EXPERIMENTAL PROCEDURES}

We used a ultra-high vacuum sputtering system with a base pressure of $1 \times 10^{-7} \mathrm{~Pa}$ for the films growth. CoFeB and Pt layers (both $3 \mathrm{~nm}$ in thickness) were deposited onto a glass substrate. We particularly used glass as our substrate because the thermal conductivity of glass is $1 \mathrm{~W} / \mathrm{m} \cdot \mathrm{K}^{24}$, much lower than that of $\mathrm{Si}(148 \mathrm{~W} / \mathrm{m} \cdot \mathrm{K})^{25}$. In doing so, we were able to heat our devices more efficiently by the microwaves injection. The grown thin-films have been patterned into micro-bar shapes having width of $32 \mu \mathrm{m}$. An on-chip waveguide made of $\mathrm{Cu}$ has been fabricated on top of each bar so that the bar became a shorted connection between the waveguide's signal and ground lines where the gap is $50 \mu \mathrm{m}$. Figure 1(a) depicts our measurement set-up and circuitary used in this study. We applied an external magnetic field along different in-plane directions and performed a series of FMR measurements by changing microwave power and frequency. All the FMR measurements presented in this paper have been carried out at a base temperature of $300 \mathrm{~K}$.

\section{RESULTS AND DISCUSSIONS}

In Fig. 1(b), we show FMR voltage signals measured at difference microwave frequency $(f)$. To extract device/materials parameters of spin transport and spin dynamics, we used the following fitting functions derived from a macrospin precession model ${ }^{26}$.

$$
\begin{gathered}
V_{\mathrm{dc}}=V_{\mathrm{sym}} \frac{\Delta H^{2}}{\left(H_{\mathrm{ext}}-H_{\mathrm{res}}\right)^{2}+\Delta H^{2}}+V_{\mathrm{asy}} \frac{\Delta H\left(H_{\mathrm{ext}}-H_{\mathrm{res}}\right)}{\left(H_{\mathrm{ext}}-H_{\mathrm{res}}\right)^{2}+\Delta H^{2}} \\
V_{\mathrm{sym}}(\phi)=\alpha I_{\mathrm{MW}} \sin 2 \phi \cdot h_{z} \cos \phi \\
V_{\text {asy }}(\phi)=\beta I_{\mathrm{MW}} \sin 2 \phi \cdot h_{y} \cos \phi
\end{gathered}
$$

Here $H_{\text {ext }}, H_{\text {res }}, \Delta H, I_{\mathrm{MW}}, \phi$ and $h_{y(z)}$ are the external dc magnetic field, the FMR field, the linewidth, the microwave current amplitude, angle defined from the bar orientation and the effective microwave fields along the $y(z)$ direction, respectively; the exact expression of parameters $\alpha$ and $\beta$ can be found in elsewhere ${ }^{26,27}$. Microwave currents in our devices induced spin currents flowing into the CoFeB layer. This mechanism ${ }^{8,12}$ exerts a damping-like torque on $\mathrm{CoFeB}$ moments and produces an effective magnetic field along the $z$ orientation. Simultaneously the same microwave currents also induced an effective field along the $y$ orientation due to classical Ampere's law. As shown in the above equations, these two effective 
fields (and therefore torques) result in voltages with different lineshapes, allowing us to identify the two torque origins independently. We first show in Fig. 1(c) $H_{\text {res }}$ versus frequency plots measured at power of $32 \mathrm{~mW}$. The Kittel formula $f=(\gamma / 2 \pi)\left[H_{\text {res }}\left(H_{\text {res }}+4 \pi M_{\mathrm{s}}\right)\right]^{1 / 2}$ was used to fit the experimental deta where $\gamma$ and $M_{\mathrm{s}}$ are the gyromagnetic ratio and the saturation magnetisation respectively; we approximate that the demagnetisation field in the $\mathrm{CoFeB}$ layer is $4 \pi M_{\mathrm{s}}$. A good agreement between the experimental data and Kittel function suggests that measured voltages in our experiments are predominantly from FMR in the CoFeB layer. One systematic change we can observe in the power dependence is that $H_{\text {res }}$ shifts to higher values when the microwave power is increased. This, by using the Kittel function, means that high power excitation in our experiments leads to a reduction of $M_{\mathrm{s}}$ due to a temperature rise in the device. We will quantitatively discuss this $M_{\mathrm{s}}$ reduction later.

Another important element in Eq. 1 is the angle dependence of $V_{\mathrm{dc}}$, i.e. $V_{\mathrm{sym}}$ and $V_{\text {asy }}$ both showing $\sin 2 \phi \cos \phi$. This symmetry is excellently demonstrated by our measured voltages shown in Fig. 2(a). These confirm that the predominant contribution of voltage generation in our experiments is from the damping- and field-like torques discussed above, excluding any other possible contributions due to microwave injection. Our CoFeB layer should most likely be amorphous, only having the shape (magnetic dipole) anisotropy from the bar dimensions. We can confirm this by showing the cos $2 \phi$-angle dependence of the $H_{\text {res }}$ in Fig. 2(b).

We next introduce our bolometric analysis. When a microwave is injected in our device, a resistive loss takes place and results in local heating. Due to the fact that sample resistance is a function of temperature, it is possible to monitor the actual device temperature via resistance. We measured our sample resistance for different microwave powers as shown in Fig. 3(a). A clear resistance increase has been measured originating in temperature rise induced by the microwaves. We scaled this temperature rise with the temperature dependence of the film resistance measured separately (Fig. 3(b)) to estimate the device temperature at a given microwave power. Figure 3(c) shows the device temperature as a function of the microwave power, which demonstrates that the temperature rise and the microwave power have a linear relationship, as expected from $Q=C \Delta T$ where $Q, C$ and $\Delta T$ are heat, specific heat capacity and temperature difference, respectively. Note here that $Q$ induced by Joule heating is proportional to microwave power in our device.

With the temperature scaled by microwave power, it is now possible to discuss magnetic 
and spin transport parameters at high temperatures. Figure 4(a) shows $M_{\mathrm{s}}$ as a function of the device temperature. We employed a phenomenological equation of $M_{\mathrm{s}}(T)=M_{\mathrm{s}}(0)[1-$ $\left.\left(T / T_{\mathrm{C}}\right)^{\alpha}\right]$ where $M_{\mathrm{s}}(0), T_{\mathrm{C}}$ and $\alpha$ are the saturation magnetisation at $T=0 \mathrm{~K}$, the Curie temperature and an exponent constant, respectively ${ }^{28}$. A best fit using this equation gives $M_{\mathrm{s}}$ of $1.59 \mathrm{~T}$ and $T_{\mathrm{C}}$ of $920( \pm 57) \mathrm{K}$, both of which show fairly good agreements with previously reported values of $\mathrm{CoFeB}$ thin-films ${ }^{28}$. To comfirm the $M_{\mathrm{s}}$ reduction by the temperature rise, we quantify the size of this anisotropy field $H_{\mathrm{u}}$ by the amplitude of oscillation $H_{\mathrm{u}}$ as shown in Fig. 4(b) and confirm that it does not vary with temperature. The fluctuation of $H_{\mathrm{u}}$ is around $2 \mathrm{mT}$ which is much smaller than the $M_{\mathrm{s}}$ reduction of about $70 \mathrm{mT}$ over the measured range. We also employed this technique to study $\theta_{\mathrm{SH}}$ of our Pt layer at high temperatures. In ST-FMR experiments on FM/NM bi-layer structures, $\theta_{\mathrm{SH}}$ can be extracted by using the following expression ${ }^{12}$.

$$
\theta_{\mathrm{SH}}=\left(\frac{V_{\mathrm{sym}}}{V_{\mathrm{asy}}}\right)\left(\frac{e \mu_{0} M_{\mathrm{s}} t d}{\hbar}\right) \frac{1}{\sqrt{1+\left(4 \pi M_{\mathrm{s}} / H_{\mathrm{res}}\right)}}
$$

Here, $e, \mu_{0}, t(d)$ and $\hbar$ are the elementary charge, the permeability in vacuum, the thickness of the $\mathrm{CoFeB}(\mathrm{Pt})$ layer and the reduced Planck constant, respectively. Related and crucial to this analysis is the power dependence of both $V_{\text {sym }}$ and $V_{\text {asy }}$ shown in Fig. 5(a). From these, a linear relationship between $V_{\text {sym }}\left(V_{\text {asy }}\right)$ and the microwave power is revealed, which is predicted by Eq. (2) and (3) - note that the spin-orbit fields are proportional to $I$, hence e.g. $V_{\text {sym }} \propto I h_{z} \propto I^{2} \propto P$. We calculated $\theta_{\mathrm{SH}}$ for each power, and the temperature dependence of $\theta_{\mathrm{SH}}$ is plotted in Fig. $5(\mathrm{~b})$. Within the measured temperature region, $\theta_{\mathrm{SH}}$ was found to be around $1.7 \times 10^{-2}$ which is consistent with reported values ${ }^{17,18,21-23}$ of $\theta_{\mathrm{SH}}$ in Pt. Another observation in $\theta_{\mathrm{SH}}$ is a slight upturn for higher temperature, i.e. about 2 $\%$ change from RT up to the highest temperature measured. This trend is very similar to $\theta_{\mathrm{SH}}$ that Isasa et al. measured in their Pt layer within lateral spin-valve devices below RT. Their $\theta_{\mathrm{SH}}$ measured in Pt increased from 0.009 at $10 \mathrm{~K}$ to 0.01 at $300 \mathrm{~K}$ which are compared to SHE originated from the intrinsic and extrinsic mechanisms. Isasa et at. concluded that the observed monotonic increase of $\theta_{\mathrm{SH}}$ with temperature is mainly due to the intrinsic SHE mechanism in $\mathrm{Pt}$, consistent of $\theta_{\mathrm{SH}}$ with others work reported earlier. The temperature dependence of $\theta_{\mathrm{SH}}$ in our study supports the conclusion of Isasa et al. in that a monotonic increase of $\theta_{\mathrm{SH}}$ is sustained even at higher than RT. It should be however noted that the rate 
of increase per temperature is small and that the overall change is comparable to the size of our error bars. From this we anticipate that there must be a non-negligible contribution from the extrinsic skew scattering mechanism in which $\theta_{\mathrm{SH}}$ is temperature-independent. Nevertheless, the magnetic and spin transport parameters discussed here demonstrate that the bolometric FMR technique can be indeed a simple yet powerful tool to study magnetism and spin transport physics at high temperatures within standardised ST-FMR techniques.

\section{SUMMARY}

In summary, we report on the new current-induced FMR technique to characterise hightemperature spin-Hall angles as well as other magnetic parameters in thin-film bi-layer devices. Using a ST-FMR device based on a CoFeB/Pt thin-film grown on a glass substrate, we have shown that it is possible to control the device temperature by changing the power of microwaves that are originally used to drive FMR. The device temperature was calculated through the resistance as a calibration parameter. We observed clear shifts of FMR peak positions for high power measurements, which is explained as a reduction of saturation magnetisation due to the temperature increase. From this, we were able to estimate the Curie temperature of our CoFeB film being $920( \pm 57) \mathrm{K}$. The temperature dependence of $\theta_{\mathrm{SH}}$ in Pt has been characterised by using the FMR voltages. We found that there is a slight increase of $\theta_{\mathrm{SH}}$ with temperature. This temperature dependence is consistent with the intrinsic mechanism of SHE, supporting the conclusion drawn by Isasa et al but also suggesting the presence of the temperature-independent skew scattering component. This simple method is widely accessible to anyone working in spin-Hall-induced ST-FMR studies in magnetic bi-layers and hence very useful to characterise spin-orbit transport physics in not only other heavy metals but also any emerging materials.

\section{ACKNOWLEDGEMENT}

We acknowledge Dr Tatsuya Nomura for his supports to this study by sample fabrication. This work is supported by JSPS Program for Fostering Globally Talented Researchers. Y. Y. and H. K. acknowledge the financial support from a Kyushu University research programme 
"PROGRESS100".

1 S. Maekawa, S. Valenzuela, E. Saitoh, T. Kimura (eds.). Spin Current. Oxford: Oxford University Press (2012). https://doi.org/10.1093/acprof:oso/9780199600380.001.0001.

2 D. C. Ralph, M. D. Stiles, Spin transfer torques, J. Magn. Magn. Mater. 320, 1190-1216 (2008). https://doi.org/10.1016/j.jmmm.2007.12.019.

3 T. Kawahara, K. Ito, R. Takemura, H. Ohno, Spin-transfer torque RAM technology: Review and prospect, Microelectron. Reliab. 52613 (2012). https://doi.org/10.1016/j.microrel.2011.09.028.

4 L. Q. Liu, C. F. Pai, Y. Li, H. W. Tseng, D. C. Ralph, and R. A. Buhrman, SpinTorque Switching with the Giant Spin Hall Effect of Tantalum, Science 336555 (2012). https://doi.org/10.1126/science.1218197.

5 S. O. Valenzuela and M. Tinkham, Direct electronic measureemnt of the spin Hall effect, Nature 442176 (2006). https://doi.org/10.1038/nature04937.

6 T. Kimura, Y. Otani, T. Sato, S. Takahashi, and S. Maekawa, RoomTemperature Reversible Spin Hall Effect, Phys. Rev. Lett. 98156601 (2007). https://doi.org/10.1103/PhysRevLett.98.249901.

7 E. Saitoh, M. Ueda, H. Miyajima, and G. Tatara, Conversion of spin current into charge current at room temperature: Inverse spin-Hall effect Appl. Phys. Lett. 88182509 (2006). https://doi.org/10.1063/1.2199473.

8 K. Ando, S. Takahashi, K. Harii, K. Sasage, J. Ieda, S. Maekawa, and E. Saitoh, Electric Manipulation of Spin Relaxation Using the Spin Hall Effect, Phys. Rev. Lett. 101036601 (2008). https://doi.org/10.1103/PhysRevLett.101.036601.

9 I. M. Miron, K. Garello, G. Gaudin, P. J. Zermatten, M. V. Costache, S. Auffret, S. Bandiera, B. Rodmacq, A. Schuhl, and P. Gambardella, Perpendicular switching of a single ferromagnetic layer induced by in-plane current injection, Nature 476189 (2011). https://doi.org/10.1038/nature10309.

10 J. Sinova, S. O. Valenzuela, J. Wunderlich, C.H. Back, and T. Jungwirth, Spin Hall effects, Rev. Mod. Phys. 871213 (2015). https://doi.org/10.1103/RevModPhys.87.1213.

11 L. Liu, R. A. Buhrman, and D. C. Ralph, Review and Analysis of Measurements of the Spin Hall Effect in Platinum, arXiv: 1111.3702 (2011). 
12 L. Q. Liu, T. Moriyama, D. C. Ralph, and R. A. Buhrman, Spin-Torque Ferromagnetic Resonance Induced by the Spin Hall Effect, Phys. Rev. Lett. 106036601 (2011). https://doi.org/10.1103/PhysRevLett.106.036601.

13 C.-F. Pai, L. Liu, Y. Li, H. W. Tseng, D. C. Ralph, and R. A. Buhrman, Spin transfer torqu devices utilizing the giant spin Hall effect of tungusten, Appl. Phys. Lett. 101122404 (2012). https://doi.org/10.1063/1.4753947.

14 Y. Niimi, H. Suzuki, Y. Kawanishi, Y. Omori, T. Valet, A. Fert, and Y. Otani, Extrinsic spin Hall effects induced by Iridium Impurities in Copper, Phys. Rev. Lett. 106, 126601 (2011). https://doi.org/10.1103/PhysRevLett.106.126601.

15 Y. Fan et al., Magnetization switching through giant spin-orbit torque in a magnetically doped topological insulator heterostructure. Nature Mater. 13699 (2014). https://doi.org/10.1038/nmat3973.

16 A. R. Mellnik et al., Spin-transfer torque generated by a topolocical insulator, Nature 511449 (2014). https://doi.org/10.1038/nature13534.

17 M. Morota, Y. Niimi, K. Ohnishi, D. H. Wei, T. Tanaka, H. Kontani, T. Kimura, and Y. Otani, Indication of intrinsic spin Hall effect in $4 \mathrm{~d}$ and $5 \mathrm{~d}$ transtion metals, Phys. Rev. B 83, 174405 (2011). https://doi.org/10.1103/PhysRevB.83.174405.

18 M. Isasa, E. Villamor, L. E. Hueso, M. Gradhand, and F. Casanova, Temperature dependence of spin diffusion length and spin Hall angle in Au and Pt, Phys. Rev. B 91024402 (2015). https://doi.org/10.1103/PhysRevB.91.024402.

19 Y. Niimi, H. Suzuki, Y. Kawanishi, Y. Omori, T. Valet, A. Fert, and Y. Otani, Extrinsic spin Hall effects measured with lateral spin valve structures, Phys. Rev. B 89054401 (2014). https://doi.org/10.1103/PhysRevB.89.054401.

20 N. Okamoto, H. Kurebayashi, T. Trypiniotis, I. Farrer, D. A. Ritchie, E. Saitoh, J. Sinova, J. Masek, T. Jungwirth, and C. H. W. Barnes, Electric control of the spin Hall effect by intervalley transitions, Nature Mater. 13932 (2014). https://doi.org/10.1038/nmat4059.

21 O. Mosendz, J. E. Pearson, F. Y. Fradin, G. E. W. Bauer, S. D. Bader, and A. Hoffmann, Quantifying Spin Hall Angles from Spin Pumping: Experiments and Theory, Phys. Rev. Lett. 104046601 (2010). https://doi.org/10.1103/PhysRevLett.104.046601.

${ }^{22}$ K. Kondou, H. Sukegawa, S. Mitani, K. Tsukagoshi and S. Kasai, Evaluation of Spin Hall Angle and Spin Diffusion Length by Using Spin Current-Induced Ferromagnetic Resonance, 
Appl. Phys. Exp. 5073002 (2012). https://doi.org/10.1143/APEX.5.073002.

23 Y. Wang, P. Deorani, X. Qiu, J.-H. Kwon, and H. Yang, Determination of intrinsic spin Hall angle in Pt, Appl. Phys. Lett. 105, 152412 (2014). https://doi.org/10.1063/1.4898593.

24 R. W. Powell, C. Y. Ho and P. E. Liley, Thermal conductivity of selected materials, NSRDS-NBS 8, 67 (1966).

25 J. C. Thompson and B. A. Younglove, Thermal conductivity of silicon at low temperatures, J. Phys. Chem. Solids 20146 (1961). https://doi.org/10.1016/0022-3697(61)90146-9.

26 D. Fang et al., Spin-orbit-driven ferromagnetic resonance, Nature Nanotechnol. 6413 (2011). https://doi.org/10.1038/nnano.2011.68.

27 H. Kurebayashi et al., An antidamping spin-orbit torque originating from the Berry curvature, Nature Nanotechnol. 9211 (2014). https://doi.org/10.1038/nnano.2014.15.

28 K.-M. Lee, J. W. Choi, J. Sok, and B.-C. Min, Temperature dependence of the interfacial magnetic anisotropy in $\mathrm{W} / \mathrm{CoFeB} / \mathrm{MgO}$, AIP Advances 7065107 (2017). https://doi.org/10.1063/1.4985720.

29 A. Hoffmann, Spin Hall Effects in Metals, IEEE Trans. Magn. 49, 5172 (2013). https://doi.org/10.1109/TMAG.2013.2262947.

30 T. Tanaka, H. Kontani, M. Naito, D. S. Hirashima, K. Yamada, and J. Inoue, Intrinsic spin Hall effect and orbital Hall effect in 4d and 5d transition metals, Phys. Rev. B 77165117 (2008). https://doi.org/10.1103/PhysRevB.77.165117. 


\section{Phu et al.: Figure 1}

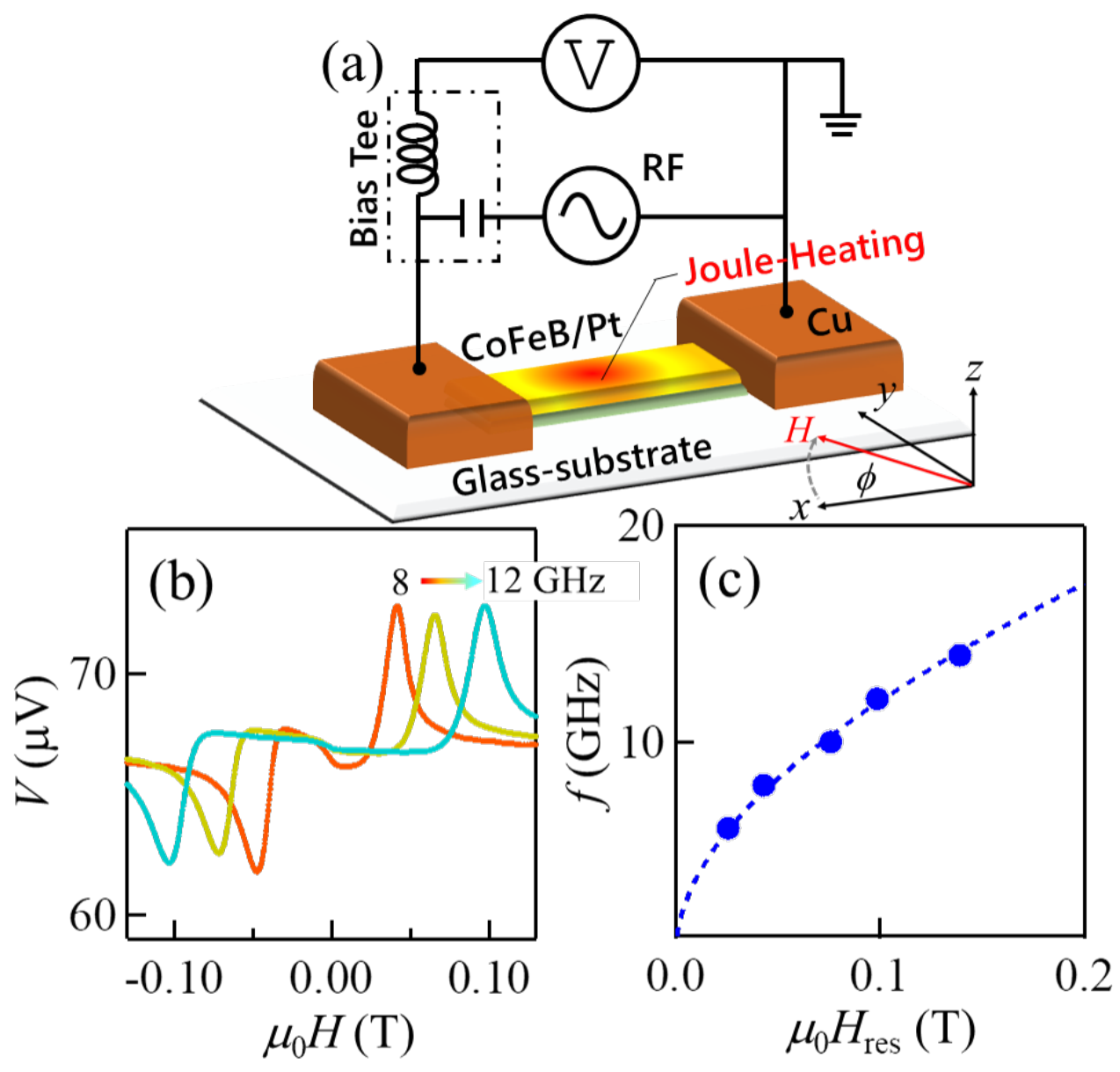

FIG. 1: (a) Schematic of the measurement set-up using this study with circuit configuration. (b) Measured voltages across the sample while sweeping the external magnetic field. We repeated this measurement by injecting a microwave current at a different frequency from 8 to $12 \mathrm{GHz}$. (c) A plot of FMR resonant fields $\left(H_{\text {res }}\right)$ vs microwave frequency $(f)$ with input-microwave power of 32 $\mathrm{mW}$. 

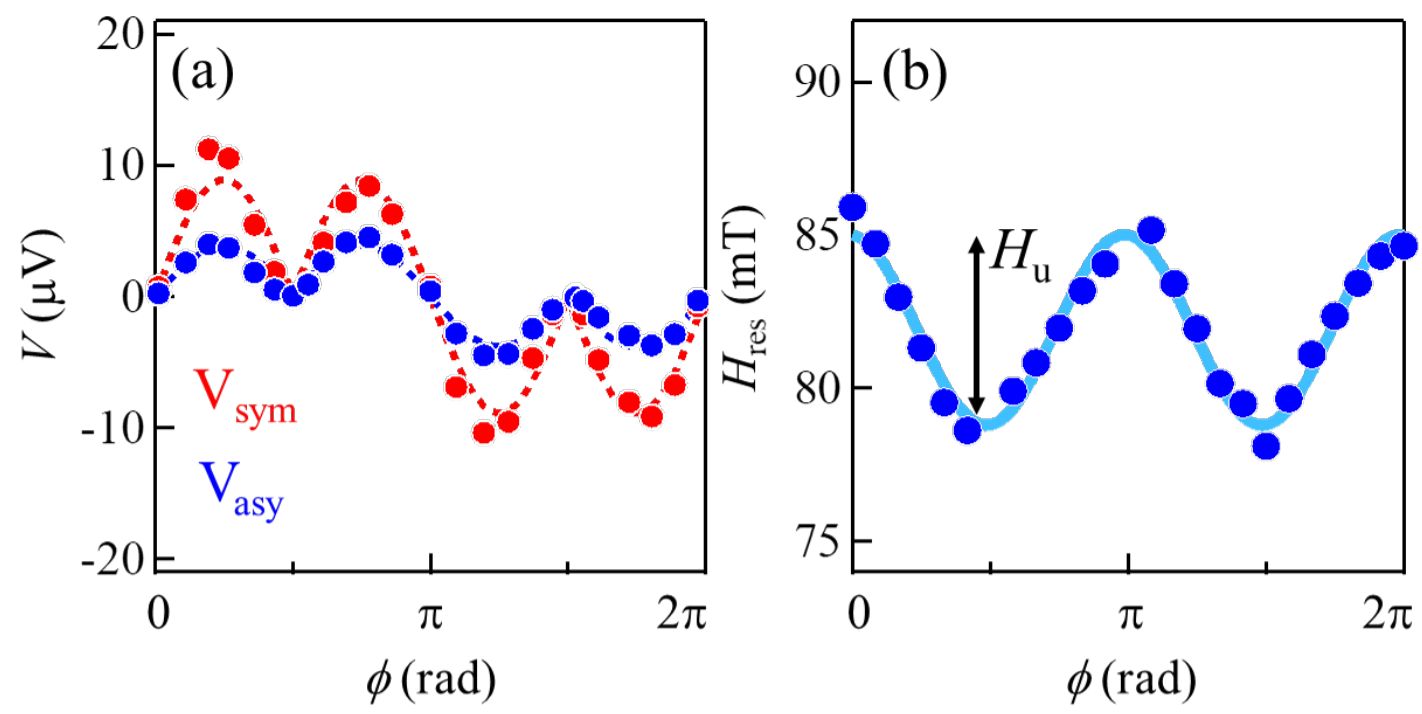

FIG. 2: (a) Angular dependence of symmetric $\left(V_{\text {sym }}\right)$ and anti-symmetric $\left(V_{\text {aym }}\right)$ Lorentzian lineshape components. Both of them show $\sin 2 \phi \cos \phi$ symmetry which agrees well with Eq. (2) and (3). (b) Angle dependence of the resonance field with input-microwave irradiation with the power of $20 \mathrm{~mW}$, which is corresponding $345 \mathrm{~K}$ (frequency is $10 \mathrm{GHz})$. From fitting using $\left(A \cos ^{2} \phi+B\right)$, anisotropic magnetic field $H_{\mathrm{u}}$ and resonance field $H_{\mathrm{res}}$ is corresponding $\mathrm{A}$ and $\mathrm{B}$, respectively.

Phu et al.: Figure 2 


\section{Phu et al.: Figure 3}
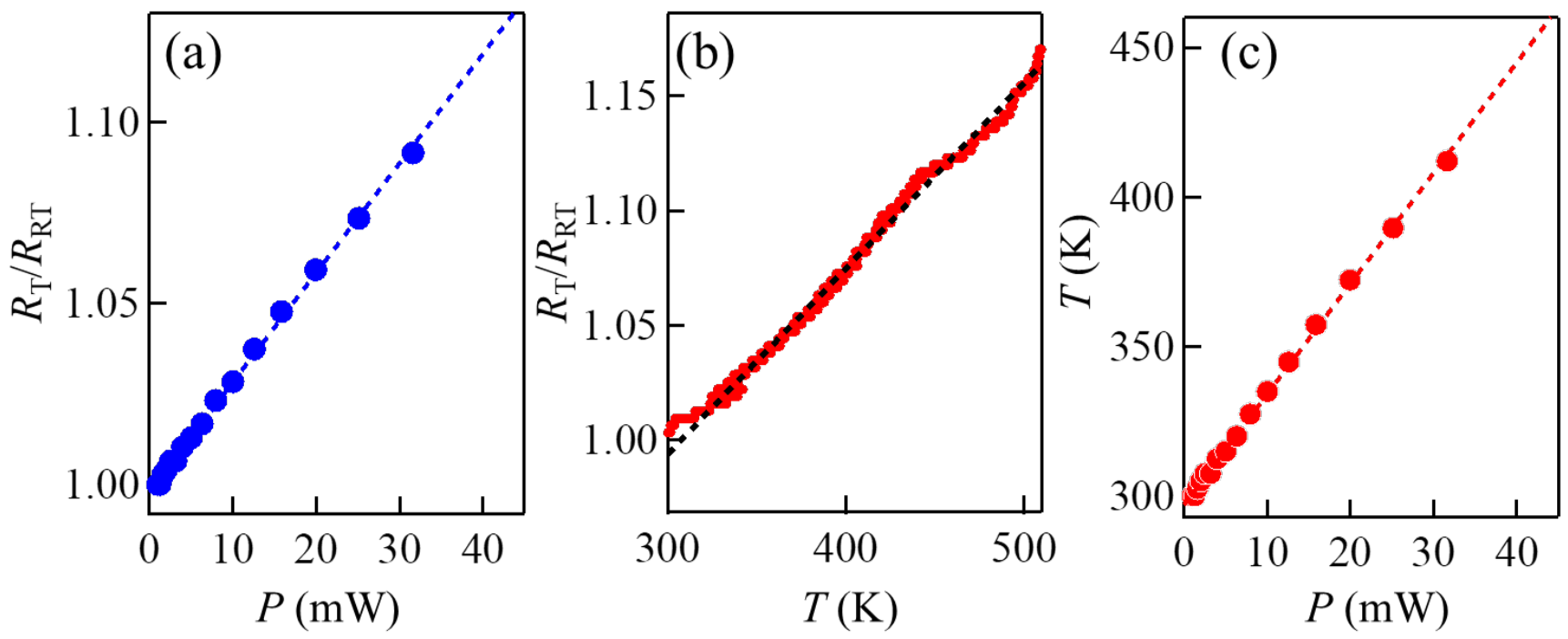

FIG. 3: (a) Device resistance measurements when injecting microwave at different powers. The resistance was normalised at the resistance with zero microwave power $\left(R_{0}\right)$. (b) Resistance measurements of the $\mathrm{CoFeB} / \mathrm{Pt}$ film at different temperatures. The resistance is normalised by the one at $T=300 \mathrm{~K}\left(R_{300 \mathrm{~K}}\right)$. (c) Scaling of temperature and microwave power by using the two sets of resistance measurements shown in Fig. 3(a) and (b). 

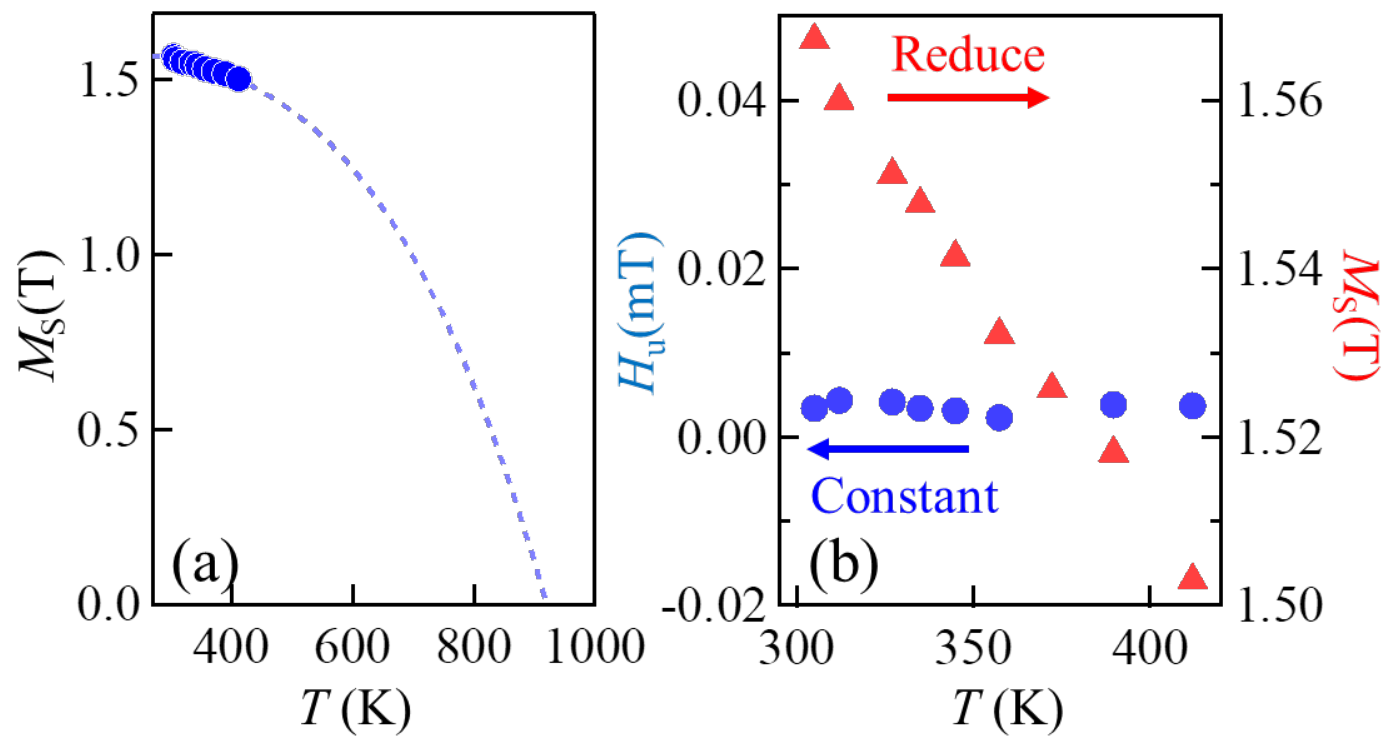

FIG. 4: (a) Saturation magnetisation $\left(M_{\mathrm{S}}\right)$ change due to an increase of device temperature. (b) Temperature dependence of the anisotropy field $H_{\mathrm{u}}$ (Blue) and saturation magnetisation $M_{\mathrm{S}}$ (Red).

Phu et al.: Figure 4 

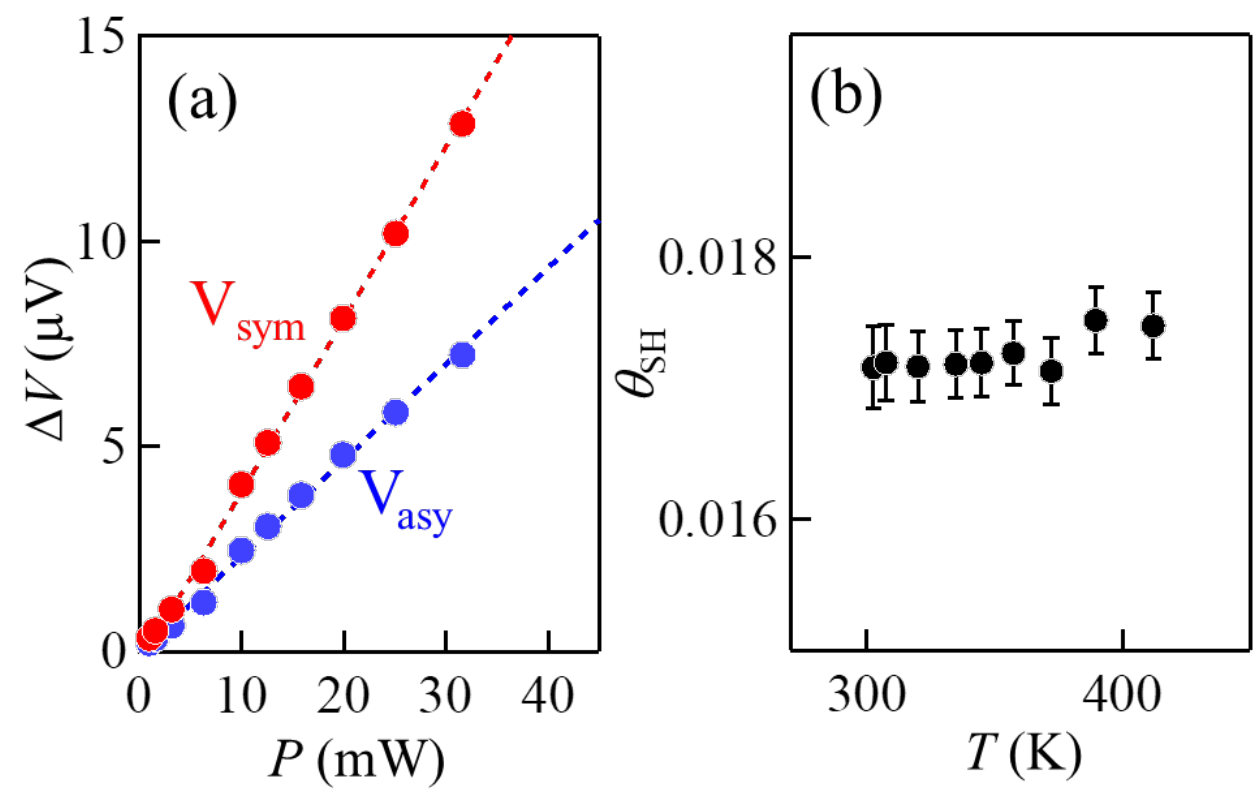

FIG. 5: (a) $V_{\text {sym }}$ and $V_{\text {asy }}$ growth by increasing microwave power. Note that the two voltage amplitudes are proportional to microwave power as expected in Eq. (2) and (3). (b) Spin-Hall angle $\left(\theta_{\mathrm{SH}}\right)$ extracted by using Eq.4 and experimental data for different temperatures. The error bars for the calculated $\theta_{\mathrm{SH}}$ represent the error involved in determining fitting function of $V_{\mathrm{dc}}$ and Kittel function in Fig.1(c)

Phu et al.: Figure 5 